\title{
Estimation of Silicon Nanocrystalline Sizes from Photoluminescence Measurements of RF Co-Sputtered $\mathrm{Si} / \mathrm{SiO}_{2}$ Films.
}

\author{
A. Ramírez-Porras ${ }^{1}$, L.F. Fonseca ${ }^{2}$, and O. Resto ${ }^{2}$ \\ ${ }^{1}$ Centro de Investigación en Ciencia e Ingeniería de Materiales and Escuela de Física, \\ Universidad de Costa Rica, San Pedro 2060, Costa Rica. \\ ${ }^{2}$ Department of Physics, University of Puerto Rico, Río Piedras, PR 00931, U.S.A.
}

\begin{abstract}
A stochastic distribution of nanocrystalline sizes model is applied to fit photoluminescence (PL) spectra of luminescent $\mathrm{Si}$ nanocrystals in a $\mathrm{Si} / \mathrm{SiO}_{2}$ matrix synthesized by $\mathrm{RF}$ co-sputtering on the top of quartz substrates. With this method, the PL spectra from a diverse set of samples can be resolved mainly as the sum of two components: a contribution from a gaussian-like distribution of sizes of quantum dots (QD) and a similar component from a distribution of quantum wires (QW). These distributions of sizes and their associated PL energies agree well with the so-called Smart Quantum Confinement model (SQC).
\end{abstract}

\section{INTRODUCTION}

The interest in the study of nanometer size silicon crystals has improved since the discovery of PL in this material more than a decade ago [1]. The increase in quantum efficiency compared to the bulk is the key point to consider this material a candidate for optoelectronic applications [2]. Despite the large number of studies dealing with the modeling of the PL mechanism (see for example reviews in Refs. [3] and [4]), a controversy is still present and no conclusive model can explain all the facets of the problem. Nevertheless, it is generally accepted that the PL emission is a consequence of a widening in the band gap due to quantum confinement in the nanocrystals [5]. Several studies confirm the presence of nanocrystals (called "quantum dots", QD) or aggregates of nanocrystals in long structures ("quantum wires", QW) in luminescent specimens [6-12]. The evidence points at the existence of statistical distributions of nanocrystal sizes mainly because the process of production of the specimens is highly stochastic [13-17]. A model by John and Singh [13] proposes that the PL characteristics should be in accordance with such distribution of sizes, at least for nanocrystals non passivated on their surface with foreign species. This is partially true for air-exposed specimens (surface passivated with oxygen), where it turns out that samples with nanocrystal mean diameter of around $3 \mathrm{~nm}$ or less do not show further dependence between PL structure and diameter [18]. A model proposed by Wolkin et al [18] takes into account the transitions from localized-to-band states that originate from the trapping of an exciton in $\mathrm{Si}=\mathrm{O}$ bonds in those nanocrystals, but assuming a sole size for them. As a result, a comprehensive model encompassing these two approaches has been proposed recently to explain the PL characteristics in porous silicon [19]. The aim of the present study is to apply the model to nanocrystalline $\mathrm{Si} / \mathrm{SiO}_{2}$ films.

\section{THEORETICAL MODELING}

The theoretical model employed here has been detailed in Ref. 19 and will be briefly described here. Consider a Gaussian distribution of QD and QW nanostructures with respective diameters 
$d_{d}$ and $d_{w}$ centered about means $d_{0 d}$ and $d_{0 w}$ and with standard deviations $\sigma_{d}$ and $\sigma_{w}$. The probability distribution of the PL process for both QD (subscript " $d$ ") and QW (subscript "w") can be obtained by the following [14]:

$$
\begin{aligned}
& P_{d}(\Delta E)=K_{d}(\Delta E)^{-3.88} \exp \left[-\frac{1}{2}\left(\frac{d_{0 d}}{\sigma_{d}}\right)^{2}\left(\frac{c_{d}^{0.72}}{d_{0 d}}(\Delta E)^{-0.72}-1\right)^{2}\right] \\
& P_{w}(\Delta E)=K_{w}(\Delta E)^{-3.16} \exp \left[-\frac{1}{2}\left(\frac{d_{0 w}}{\sigma_{w}}\right)^{2}\left(\frac{c_{w}^{0.72}}{d_{0 w}}(\Delta E)^{-0.72}-1\right)^{2}\right]
\end{aligned}
$$

where $K_{d, w}$ are normalization constants, and $\Delta E=c_{d, w} / d^{1.39}$ is the quantum confinement energy ( $c_{d, w}$ are their associated constants) [20]. $\Delta E$ is related with the PL energy ( $h v$ ), the bulk silicon gap $\left(E_{g} \sim 1.15 \mathrm{eV}\right)$ and the exciton binding energy $\left(E_{b} \sim 0.15 \mathrm{eV}\right)$ by:

$$
\Delta E=h v-\left(E_{g}-E_{b}\right) \approx h v-1(\mathrm{eV})
$$

The PL spectrum shape must be a weighted addition of these two components plus a contribution from the localized-to-band state originated from an exciton trapped in $\mathrm{Si}=\mathrm{O}$ bonds when the nanocrystal size is lesser than around $3 \mathrm{~nm}$, as mentioned above. The calculations of electronic states in nanocrystal silicon exposed to air identify three important zones in the exciton recombination process [18]. The first (zone I), for sizes larger than $3 \mathrm{~nm}$, is characterized by band-to-band transitions, and so the PL spectra is directly related with the band gap, which in turn is a function of the crystallite size. The second (zone II, sizes between 3 and $2 \mathrm{~nm}$ ) is a localized state to valence band transitions zone. This state seems to be a p-state in the Si atom of the $\mathrm{Si}=\mathrm{O}$ bond. In this zone, the relation between PL spectrum and crystallite size is weaker than for zone I. The last (zone III, sizes smaller than $2 \mathrm{~nm}$ ) is characterized by localized-to-localized states, one belonging to $\mathrm{Si}$ atom and the other to $\mathrm{O}$ atom in the $\mathrm{Si}=\mathrm{O}$ bond. In this case, no functionality between PL spectrum and crystallite size is observed. This particular effect was also reported by others [21], and possible effects of dangling bonds recombinations were ruled out [20].

We therefore assume a Gaussian contribution of localized-to-band recombinations, valid for zones II and III, in the form:

$$
P_{l o c}(\Delta E)=K_{l o c} \exp \left[-\frac{1}{2}\left(\frac{(\Delta E)-(\Delta E)_{0}}{\sigma_{l o c}}\right)^{2}\right]
$$

where $K_{l o c}$ is a normalization constant, $(\Delta E)_{0}$ the mean of the distribution and $\sigma_{l o c}$ its standard deviation.

\section{EXPERIMENTAL PROCEDURE AND METHODS}

The $\mathrm{Si}-\mathrm{SiO}_{2}$ films were synthesized on a 6-inch long quartz substrate by RF co-sputtering. The deposition was performed in a high vacuum chamber previously evacuated to $1.0 \times 10^{-7}$ Torr and under an argon pressure of $2.0 \times 10^{-2}$ Torr at $115^{\circ} \mathrm{C}$ substrate temperature. The target configuration included a $\mathrm{SiO}_{2}$ disk (6 inches in diameter) and a piece of Si wafer (area $\sim 0.1 \mathrm{in}^{2}$ ) 
mounted on top of the $\mathrm{SiO}_{2}$ target disk just below one end of the substrate deposition area. The sputtering time was 12 hours for sample 1 and 3 hours for sample 2. The position of the Si wafer on the target was used to control the size of silicon nanoparticles. The films were thereafter annealed in $\mathrm{N}_{2}$ at $1100^{\circ} \mathrm{C}$ for 30 minutes. The resulting films are $5 \times 0.2$ inches in area and a thickness of around 7.0 microns (sample 1) and 1.7 microns (sample 2). Size and density of silicon nanocrystals vary continuously along the length of the films. Photoluminescence (PL) measurements were performed at room temperature by the use of a quasi back-scattering geometry with a $514.5 \mathrm{~nm}$ line of a $\mathrm{Ar}^{+}$laser as light source. The spectra were dispersed by a TRIAX 320 spectrometer and detected by a cooled photomultiplier with GaAs photocathode.

Once the PL spectra were recorded, a fitting procedure software was employed to first determine the statistical parameters of Eqs. (1) and (2), assuming initially that only QD and QW were responsible for the obtained PL. With the mean diameters extracted from the fitting method, it was decided whether the actual PL spectrum belonged to zone I, II or III mentioned in the preceding section. If zone I was selected, then no Localized-to-Band transition was considered and the process stopped. If zone II was selected, a new fitting process was performed with the additional presence of localized states. None of our samples were attributed to zone III due to the obtained values of $d$. Statistical parameters of Eqs. (1), (2) and (4) were therefore extracted.

\section{RESULTS AND DISCUSSION}

Figure 1 shows the normalized PL spectra for two samples excited with the green $514.5 \mathrm{~nm}$ line of an $\mathrm{Ar}^{+}$laser. The films were divided in 50 equal segments and designated a number. Number 1 was assigned to $\mathrm{SiO}_{2}$ rich end and 50 to the Si rich end of the film. Each segment has different nanocrystal density and size. PL spectra are broad and shift to higher energies as one moves towards lower position labels (that is, from $\mathrm{Si}$ rich to $\mathrm{SiO}_{2}$ rich). The behavior suggests that nanocrystal sizes decrease for lower position numbers. The statistical results from the fittings are presented in Table 1 for the whole set of studied samples. Typical fits from PL
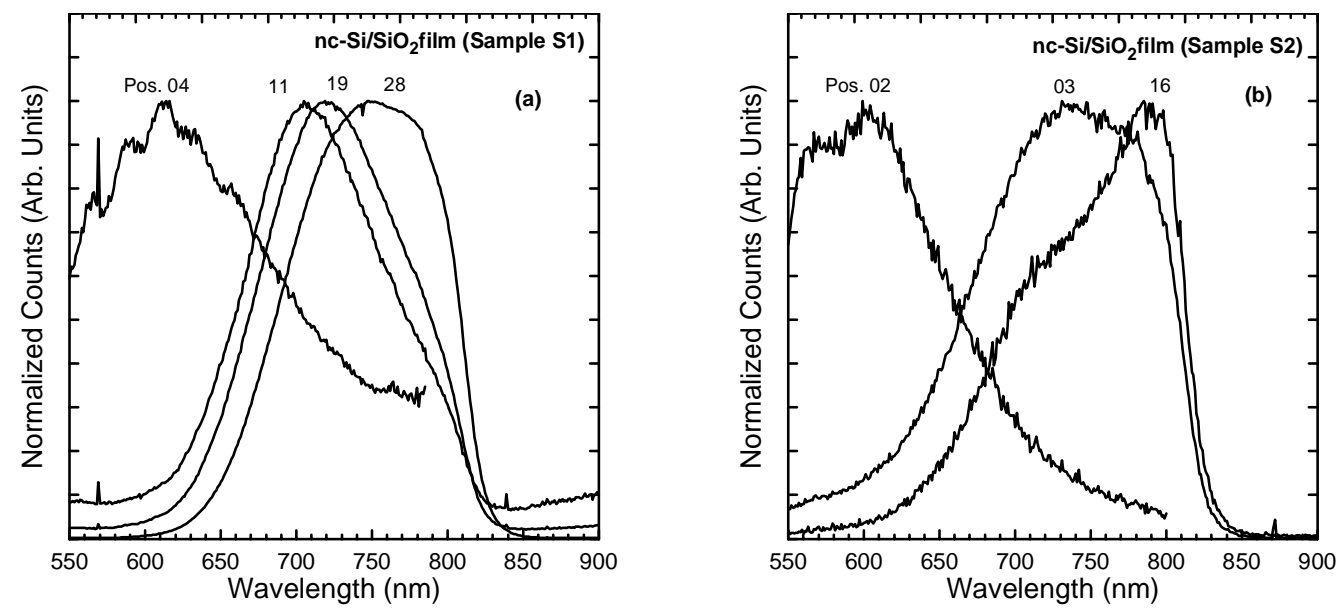

Figure 1. Normalized size-dependent PL spectra from silicon nanocrystals embedded in silicon dioxide for two samples: sample S1 (a), and sample S2 (b). The excitation wavelength was the $514.5 \mathrm{~nm} \mathrm{Ar}^{+}$laser. The different spectra for each plot correspond to different positions along the film, as marked. Lower position numbers are $\mathrm{SiO}_{2}$ rich, and higher position numbers are Si rich. 
Table I: Statistical parameters extracted from the fitting procedure. $d_{0 d}, \sigma_{d}, d_{0 w}$ and $\sigma_{w}$ are related with the QD and QW crystallites, respectively. $E_{0, l o c}$ and $\sigma_{0, l o c}$ are the actual mean energy and the standard deviation of the expected Localized-to-Band transitions, respectively.

\begin{tabular}{|ccccccccc|}
\hline $\begin{array}{c}\text { Sample } \\
\text { Label }\end{array}$ & Position & Zone & $\boldsymbol{d}_{\boldsymbol{l d}}(\mathbf{n m})$ & $\sigma_{\boldsymbol{d}}(\mathbf{n m})$ & $\boldsymbol{d}_{\boldsymbol{o}_{\boldsymbol{w}}}(\mathbf{n m})$ & $\sigma_{\boldsymbol{w}}(\mathbf{n m})$ & $\boldsymbol{E}_{\text {o,loc }}(\mathbf{e V})$ & $\sigma_{0, \text { loc }}(\mathbf{e V})$ \\
\hline S1-04 & 04 & II & 2.01 & 0.37 & 3.71 & 0.90 & 1.95 & 0.18 \\
S1-11 & 11 & I & 2.88 & 0.23 & 3.79 & 0.40 & - & - \\
S1-19 & 19 & I & 3.02 & 0.24 & 4.05 & 0.33 & - & - \\
S1-28 & 28 & I & 3.14 & 0.28 & 4.24 & 0.26 & - & - \\
\hline S2-02 & 02 & II & 2.22 & 0.10 & 3.35 & 0.39 & 2.06 & 0.08 \\
S2-03 & 03 & I & 2.98 & 0.44 & 4.30 & 0.20 & - & - \\
S2-07 & 07 & I & 3.23 & 0.38 & 4.34 & 0.20 & - & - \\
S2-11 & 11 & I & 3.23 & 0.39 & 4.37 & 0.22 & - & - \\
S2-16 & 16 & I & 3.12 & 0.39 & 4.29 & 0.21 & - & - \\
\hline
\end{tabular}

spectra belonging to zones I and II are shown in figure 2. Notice an agreement in the reduction of nanocrystal sizes for blue-shifted spectra. QD contribution has stronger peaks than QW, which can suggest that QD are more likely to get formed than QW.

From the data of $d_{0 d}$ it is possible to calculate the mean quantum confinement energy $\Delta E$ and the peak PL energy from $h v \approx \Delta E+1(\mathrm{eV})$. Figure 3 shows the plot initially reported by Wolkin's group [18] with added results from Ramirez-Porras and Weisz [19] and results from this work. The figure does not include standard deviation bars (corresponding to the $\sigma$ values) to avoid confusion. The curves correspond to the theoretical cases deduced for spherical crystallites, and therefore we only show the results for QD (QW mean diameters lie outside the plotted region in any case, and its contribution is a subject of a further study not included here). The agreement between the experimental points and the theoretical curves is notable.
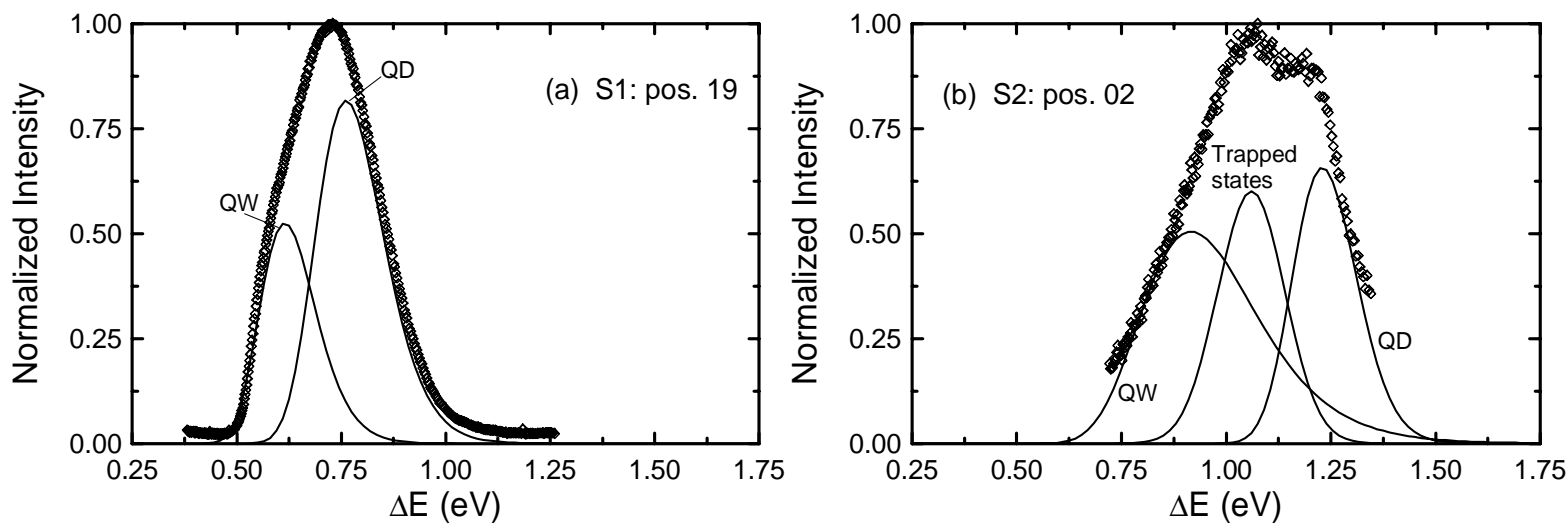

Figure 2. PL spectra as a function of $\Delta E$ for two cases: Sample $S 1$, position 19 (a) and Sample $\mathrm{S} 2$, position 02 (b). In (a), only the contributions of QD and QW are sufficient to fit the experimental data, which is characteristic of zone I. In (b), a gaussian peak (attributed to trapped states and characteristic of zone II) is necessary to fit the data. Statistical parameters of both cases are listed in Table 1. 


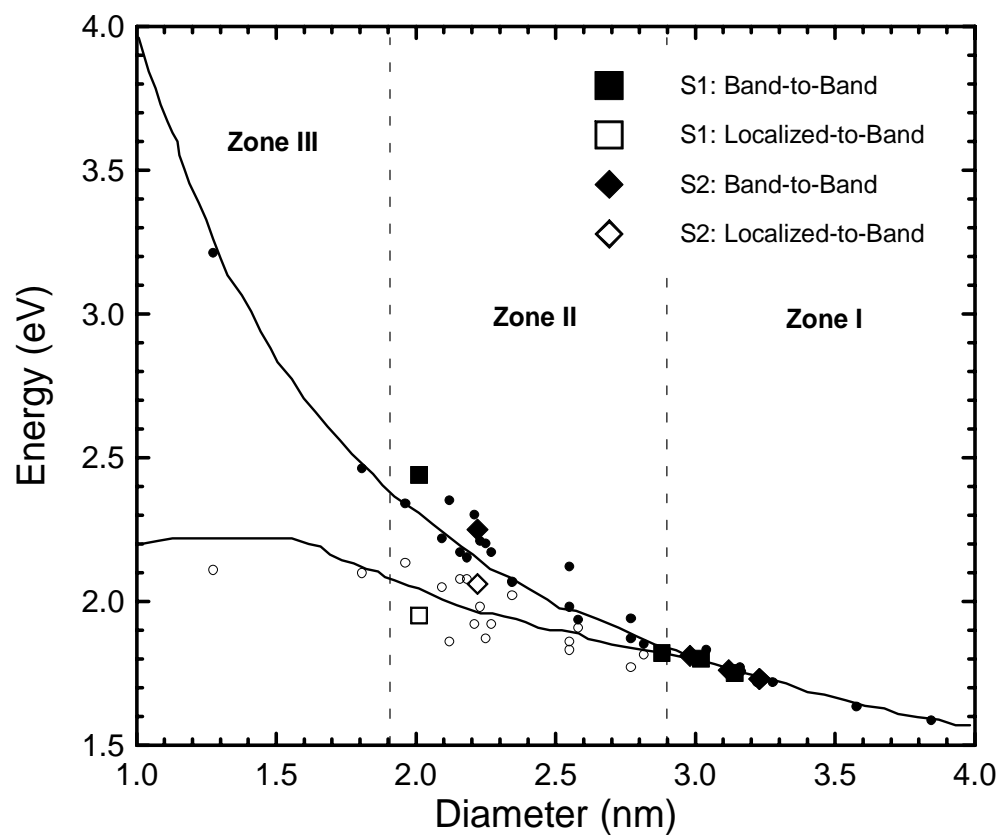

Figure 3. PL energy as a function of crystallite diameter for theory (solid lines) and experimental results. The small open and full circles are results reported in Refs. [18-19]. Open and full squares are our results. The full symbols consider radiative Band-to-Band recombinations, whereas the empty symbols represent Localized-to-Band recombinations.

In figure 3, full symbols represent Band-to-Band transitions where the PL emission is explained by pure quantum confinement. Empty symbols are Localized-to-Band transitions explained by the presence of $\mathrm{Si}=\mathrm{O}$ bonds. The major part of cases belong to zone I, and only two fall deeply into zone II, precisely the lower position number ones.

\section{CONCLUSIONS}

A unified model accounting for the $\mathrm{Si}=\mathrm{O}$ trapped state approach and the stochastic distribution of nanocrystal sizes approach has been successfully applied on $\mathrm{Si} / \mathrm{SiO}_{2}$ films. The results for QD are in good agreement with previous published theoretical results and indicate that contributions from Band-to-Band recombinations are important in all samples as well as Localized-to-Band recombination in samples with smaller nanocrystal sizes. The presence of localized states in the $\mathrm{SiO}_{2}$ rich nanocrystals is therefore evidenced. These results support the stochastic extended Smart Quantum Confinement model.

\section{ACKNOWLEDGEMENTS}

This work is based upon work supported in part by the U. S. Army Research Office under grant number 41002-MS-DPS and NASA grant No. NCC5-518. One of us (A.R-P) wishes to acknowledge partial support from the Comisión Nacional de Investigaciones Científicas y 
Tecnológicas (CONICIT), the Ministerio de Ciencia y Tecnología of Costa Rica and the Fundación de la Universidad de Costa Rica para la Investigación (FUNDEVI).

\section{REFERENCES}

1. L.T. Canham, Appl. Phys. Lett. 57 (1990) 1046.

2. L. Tsybeskov, MRS Bulletin 23 (1998) 33.

3. O. Bisi, S. Ossicini and L. Pavesi, Surface Review Reports 38 (2000) 1.

4. P. Bettotti, M. Cazzanelli, L. Dal Negro, B. Danese, Z. Gaburro, C.J. Oton, G. Vijaya Prakash and L. Pavesi, J. Phys.: Condens. Matter 14 (2002) 8253.

5. C. Delerue, G. Allan, and M. Lannoo, Journal of Luminescence 80 (1999) 65.

6. Z. Sui, P.P. Leong, I.P. Herman, G.S. Higashi, and H. Temkin, Appl. Phys. Lett. 60 (1992) 2086.

7. H. Münder, C. Andrzejak, M.G. Berger, U. Klemradt, H. Lüth, R. Herino, and M. Ligeon, Thin Solid Films 221 (1992) 27.

8. R.K. Soni, L.F. Fonseca, O. Resto, S.Z. Weisz, and S. Tripathy, Mat. Res. Soc. Symp. Proc. 571 (2000) 235.

9. R.K. Soni, L.F. Fonseca, O. Resto, M. Buzaianu and S.Z. Weisz, Journal of Luminescence 83-84 (1999) 187.

10. M.H. Nayfeh, Z. Yamani, O. Gurdal, and A. Alaql, Mat. Res. Soc. Symp. Proc. 536 (1999) 191.

11. V. Lehmann, B. Jobst, R. Muschik, A. Kux, and V. Petrova-Koch, Jpn. J. Appl. Phys. 32 (1993) 2095.

12. Naudon, P. Goudeau, and V. Vezin, in: J.C. Vial and J. Derrien (Eds.), Porous Silicon Science and Technology (Les Editions de Physique, Paris, 1995).

13. G.C. John and V.A. Singh, Phys. Rev. B 50 (1994) 5329.

14. H. Elhouichet, B. Bessaïs, O. Ben Younes, H. Ezzaouia, and M. Oueslati, Thin Solid Films 304 (1997) 358.

15. H.S. Mavi, B.G. Rasheed, R.K. Soni, S.C. Abbi, and K.P. Jain, Thin Solid Films 397 (2001) 125.

16. H. Yorikawa, and S. Muramatsu, Journal of Luminescence 87 (2000) 423.

17. S. Miyazaki, A. Mouraguchi, and K. Shiba, Thin Solid Films 297 (1997) 183.

18. M.V. Wolkin, J. Jorne, P.M. Fauchet, G. Allan, and C. Delerue, Phys. Rev. Lett. 82 (1999) 197; Mat. Res. Soc. Symp. Proc. 536 (1999) 185.

19. A. Ramírez-Porras and S.Z. Weisz, Surf. Sci. 515 (2002) 18

20. C. Delerue, G. Allan, and M. Lannoo, Phys. Rev. B 48 (1993) 11024.

21. Y. Kanemitsu, H. Mimura, T. Matsumoto, and T. Nakamura, Journal of Luminescence 72-74 (1997) 344.

22. C. Delerue, G. Allan, and M. Lannoo, Journal of Luminescence 80 (1999) 65. 